$\bullet 157$

EFFECTS OF ACUTE HYPOXIA ON THE CELLULAR ELECTRICAL ACTIVITY OF NEONATAL AND ADULT PURKINJE FIBERS (PF). Steven M. Yabek, Rinya Kato, Bramah N. Singh, Dept. of Pediatrics, University of New Mexico, A7buquerque, NM.

We previously described that action potential (AP) parameters from neonatal $(N)$ ventricular myocardium are more resistant than adult $(A)$ APs to acute hypoxia $(H)$. In this study, we used standard microelectrode techniques to evaluate the effects of acute $H$ ( $p_{2}=30$ torr $)$ on $A(n=10)$ and $N(3-12$ days old $)(n=11)$ canine PF APs and PF arrhythmogenesis. PFs were paced at $1 \mathrm{~Hz}$. Compared to $A$, control AP from N PF had significantly $(s)(p<0.05)$ lower values for $A P$ amplitude (APA), Vmax, AP duration at $50 \%$ repolarization (APD50), APD90 and effective refractory period (ERP). After $30 \mathrm{~min}$. of acute hypoxic superfusion, $N$ PF showed s greater reductions than $A P F$ in $\operatorname{Vmax}(-20 \%$ vs $-9 \%)$, APD50 $(-18 \%$ vs $-8 \%)$ and APD $90(-13 \%$ vs $-4 \%)$. Changes in APA, maximum diastolic potential (MDP) and ERP did not differ. A11 N AP parameters (except MDP) were $s$ reduced after $H$. Reoxygenation for 30 min. partially restored N AP indices. When pacing was stopped during control, no A or N PF had spontaneous activity (sa) for $15 \mathrm{sec}$. $H$ produced sa in 0 of $10 \mathrm{~A}$ and 2 of $11 \mathrm{~N}$ PF. The sa increased during early reoxygenation ( 1 of 10 in $A ; 4$ of 11 in $N$ ) but was abolished in all after $30 \mathrm{~min}$. The $H$ and reoxygenation related ectopic sa was due to both enhanced automaticity and reentry, the Tatter probably induced by the decreases in Vmax (conduction velocity), APD and ERP. In summary, N PF have an enhanced sensitivity to acute $H$. The arrhythmogenic effects of $H$ in-vitro may explain the $H$ and reperfusion related arrhythmias seen clinically in some children.

\section{$\dagger 158$} AMIODARONE (AM) ADVERSELY AFFECTS SINUS NODE (SN) of New Mexico, Albuquerque \& Wadsworth VA Hospital, L.A. $A M$ is being used more frequently to control "resistant" As a Class III agent, AM supposedly acts by uniformly prolonging action potential (AP) duration and refractoriness. Because sinus slowing is observed clinically, we evaluated the acute effects of $0.68-34 \mathrm{\mu g} / \mathrm{ml}$. AM on $\mathrm{SN}$ pacemaker
activity using rabbit SN preparations and standard microelectrode techniques. Data were collected only from true SN pacemaker cells impaled continuously throughout the study. AM produced significant $(p<0.05)$ concentration dependent decreases in spontaneous sinus rate $(-6 \%$ at $6.8 \mu \mathrm{g} / \mathrm{ml} ;-12 \%$ at $34 \mu \mathrm{g} / \mathrm{ml})$, AP amplitude $(-15 \%$ at $34 \mathrm{\mu g} / \mathrm{ml})$ and rate of phase 4 diastolic depolarization $(-14 \%,-18 \%,-27 \%$ at $0.68,6.8 \& 34 \mathrm{\mu g} / \mathrm{ml}$ respective1y). Maximum diastolic \& threshold potentials and Vmax also decreased sigmum diastolic \& threshold potentials and $\max$ also decreased nificantly. Since AP duration did not increase, the fall in
spontaneous rate was related solely to AM induced decreases in spontaneous rate was related solely to AM induced decreases in
automaticity. Corrected SNRT and SACT were increased markedly by AM. In 4 preparations, SN exit or entrance block developed on prolonged exposure. The AM induced changes could not be reversed despite superfusion in AM-free medium for up to $1.5 \mathrm{hrs}$. Rather, SN function deteriorated further. AMs Class III actions cannot explain its effects on the SN. Since the automaticity and upstroke of SN pacoker activity are dependent upon the slow inward Catt current ( $i$ ), AM may also possess Class IV (verapamilward Catt current (i $\left.i_{i}\right)$, AM may also possess Class $1 \mathrm{v}$ (ver

159 INDOMETHACIN (I) THERAPY IN PREMATURE INFANTS OF OLD POSTNATAL AGE ( $\geq 8$ WEEKS). T.F. Yeh, B. Achanti, J. Stubb, S. Pyati, R.S. Pildes.

Dept. of Ped. Previous studies have shown plasma I level and ductus closure and between clearance of plasma $I$ and postn. age; it is suggested that failure of ductus respons to $\mathrm{I}$ in some infants of old postnatal age may have been due to low plasma $I$. To test this hypothesis, 5 infants with postn. age $\geq 8$ wks were given iv I $0.3 \mathrm{mg} / \mathrm{kg} \mathrm{q} 6 \mathrm{hr}$ for 4 doses in an attemp to maintain high plasma I. All infants had sign. PDA (clinical cardiovascular distress score (CVD) $>3$ and Echo LA/AO >1.3) at time of study. The mean $\pm S . D$. birth wt was $969 \pm 209 \mathrm{gm}$; Gest. age 29 I $29 \pm 1.4$ wks, Postn. age $65.2 \pm 4.3$ days. Plasma samples \begin{tabular}{lccccc}
\multicolumn{5}{c}{ (Hrs post-study) } \\
P1asma I & $\frac{1}{2}$ & 4 & 8 & 16 & 24 \\
(ng/m1) & $828 \pm 81$ & $350 \pm 90$ & $590 \pm 102$ & $570 \pm 88$ & $370 \pm 96$ \\
Echo:LA/AO & $1.48 \pm 0.29$ & $1.51 \pm 0.30$ & $1.50 \pm 0.27$ & $1.49 \pm 0.32$ & $1.49 \pm 0.28$ \\
CVD score & $5.2 \pm 1.2$ & $4.8 \pm 1.1$ & $5.1 \pm 1.2$ & $5.1 \pm 1.0$ & $5.0 \pm 1.1$
\end{tabular} None of the infants responded to $I$ with ductus closure in spite of maintenance of plasma I concentration above presumptive therapeutic level $(250 \mathrm{ng} / \mathrm{ml}$ at $24 \mathrm{hr}$ post-dosing). There was no significant changes in LA/AO and CVD score. However, significant decreases in urine output (from $3.1 \pm 0.7$ to $2.1 \pm 0.6 \mathrm{ml} / \mathrm{kg} / \mathrm{h}, \mathrm{p}$ $<0.01)$ and $\mathrm{FENa}(1.5 \pm 0.4$ to $1.0 \pm 0.3 \%, \mathrm{p}<0.05)$ were seen after the study. This study suggests that failure to I therapy in infant of old postn. age may not relate to low plasma I level and that a primary histological anomaly of the ductus independent of $P G$ might be present in these infants.
160 RAPID ATRIAL PACING-INDUCED INFRA-HIS CONDUCTION BLOCR Ming-Lon Young, Henry Gel band, Augustin Castellanos, Grace S. Wolff. Univ of Miami, Jackson Memorial Hosp, Miami. ound to have pacing-induced infra-His conduction block (B). In 2 pts it occurred following a preceding block (B). In 2 pts it occurred following a preceding long cycle as well as a peceding short-to-long sequence cycle length (CL) change. In one of them the infrahis $B$ initiated a short sequence of $2: 1$ infra-His $B$. In 5 pts it occurred with an abrupt shortening of the
coupling $\mathrm{H}-\mathrm{H}$ (in one pt it simulated type II 2nddegree $A V B)$. In 2 pts the pacing-induced infra-His $B$ simulated type II 2nd-degree AV block but the initiating events were not recorded. Rapid atrial pacinginduced infra-His $B$ in children is a rare phenomenon. in many cases it is associated with pacing-induced In many cases it is associated with pacing-induced infra-His conduction delay. The occurrence of either as a preceding short-to-long sequence $C L$ change that can lengthen the refractoriness of the His-Purkinje system (HPS), (2) an abrupt shortening of the coupling $\mathrm{H}-\mathrm{H}$ interval that exceeds the refractory period of the HPS, (3) a first impulse that results in an infra-His $B$ initiating a series of $2: 1$ infra-His $B$ due to a self-perpetuation sequence of events, (4) a lst impulse that results in infra-His conduction delay initiating a series of $1: 1$ infra-His delay due to repetitive retrograde concealed conduction.

RVVRSED ALTERNATING WENCKEBACH PERIODICITY

1161 MingCastellanos, Grace S. Wolff, Univ of Miami, Jackson Memorial Hosp, Dept of Pedi Cardiology, Miami. Alternating Wenckebach periodicity (AWP) is defined as an pattern of 2:1 AV block associated with progressive lengthening of the conducted impulses with termination in a higher degree of block. We found another phenomenon in 5 children during atrial pacing which is phenomenon in 5 children during atrial pacing which is the reverse of this pattern (RAWP) and has not been pattern of $2: 1 \mathrm{AV}$ block, there is progressive shortening of the conducted impulses with termination in a lower degree of block. The mechanism can be explained by a one-level block model. It is formed by a series of self-perpetuating events: (1) The lst beat of the RAWP is coupled to a preceding impulse with a short coupling interval (CI) and results in a long (the longest) delay. (2) With a long preceding delay the 2nd beat of the RAWP has a short (the shortest) CI falling within the effective refractory period of the AVN (ERP-AVN) and results in block. (3) Therefore the $3 \mathrm{rd}$ beat is actualiy coupled to the lst beat with a long CI and results in a shorter delay. (4) The 4 th beat also has long $C I$, which, if still shorter than the ERP-AVN results in a sequence of $2: 1$ block in which the conducted impulses showing progressive shorwhich the conducted impulses showing progressive shorbeat exceeds the ERP-AVN and, thereby, conducts. 162 MULTILEEL AV BLOCK Ming=Lon Yos농, of Miami, Jackson Memorial Hospital, Department of Pediatric Cardiology, Miami, Florida.

Six pediatric patients (pts) exhibiting atrial pacing-induced repetitive block (B) are presented. Analysis of the conduction patterns satisfy the requisites for multilevel AV B. I) DOCUMENTED 2 LEVEL B: Two pts exhibited an uppex level AVN Wenckebach periodicity (WP) and a lower level 2:1 infra-His B. 2) DEDUCED 2 LEVEL B IN THE AVN: TWo pts had 2:1 AVN B terminated by $3: 1$ B (alternating WP). With group 1 serving as a model, it is postulated that in this group the 2 sites AVN WP and a lower level 2:1 AVN B. One pt had an AVN WP terminated by $3: 1 \mathrm{~B}$. This was followed by 2 an AVN WP terminated by $3: 1$ B. This was fol series of $2: 1$ B, suggesting simultaneous WP at 2 AVN
levels. 3) DEDUCED 3 LEVEL B IN THE AVN: One pt had levels. 3) DEDUCED 3 LEVEL B IN THE AVN: One pt had
$2: 1$ AVN B with progressive prolongation of the conducted $\mathrm{AH}$ terminating in 2 series of $3: 1 \mathrm{~B}$ in which the AH following the lst 2 blocked atrial beats was not the shortest while that following the 2nd was. With previous groups serving as models, it is postulated that there is a 3 level $B$ inside the AVN: an upper level AVN WP, a middle level AVN WP of and a lower level 2:I AVN B. In summary, we have for the first time, described multilevel $B$ within the AVN in the pediatric pt. 